\title{
Corrigendum
}

\section{Corrigendum to "Plant Leaves Extract Irrigation on Wound Healing in Diabetic Foot Ulcers"}

\author{
Muthu Srinivasan Jayalakshmi, ${ }^{1}$ P. Thenmozhi, ${ }^{2}$ and R. Vijayaraghavan ${ }^{1}$ \\ ${ }^{1}$ Saveetha Institute of Medical and Technical Sciences, Chennai, India \\ ${ }^{2}$ Saveetha College of Nursing SIMATS, Chennai, India \\ Correspondence should be addressed to Muthu Srinivasan Jayalakshmi; sekarji2003@gmail.com \\ Received 31 July 2021; Accepted 31 July 2021; Published 14 August 2021 \\ Copyright (c) 2021 Muthu Srinivasan Jayalakshmi et al. This is an open access article distributed under the Creative Commons \\ Attribution License, which permits unrestricted use, distribution, and reproduction in any medium, provided the original work is \\ properly cited.
}

In the article titled "Plant Leaves Extract Irrigation on Wound Healing in Diabetic Foot Ulcers" [1], there were errors in Figure 1, where "Follow-up-200" and "Analysis174 " should be corrected to "Follow up-173" and "Analysis-
160 ," respectively. Additionally, a number of subheadings were missing in Tables 1 and 4 . The authors confirm that this does not affect the results or conclusions of the article, and the corrected figure and tables are as follows. 


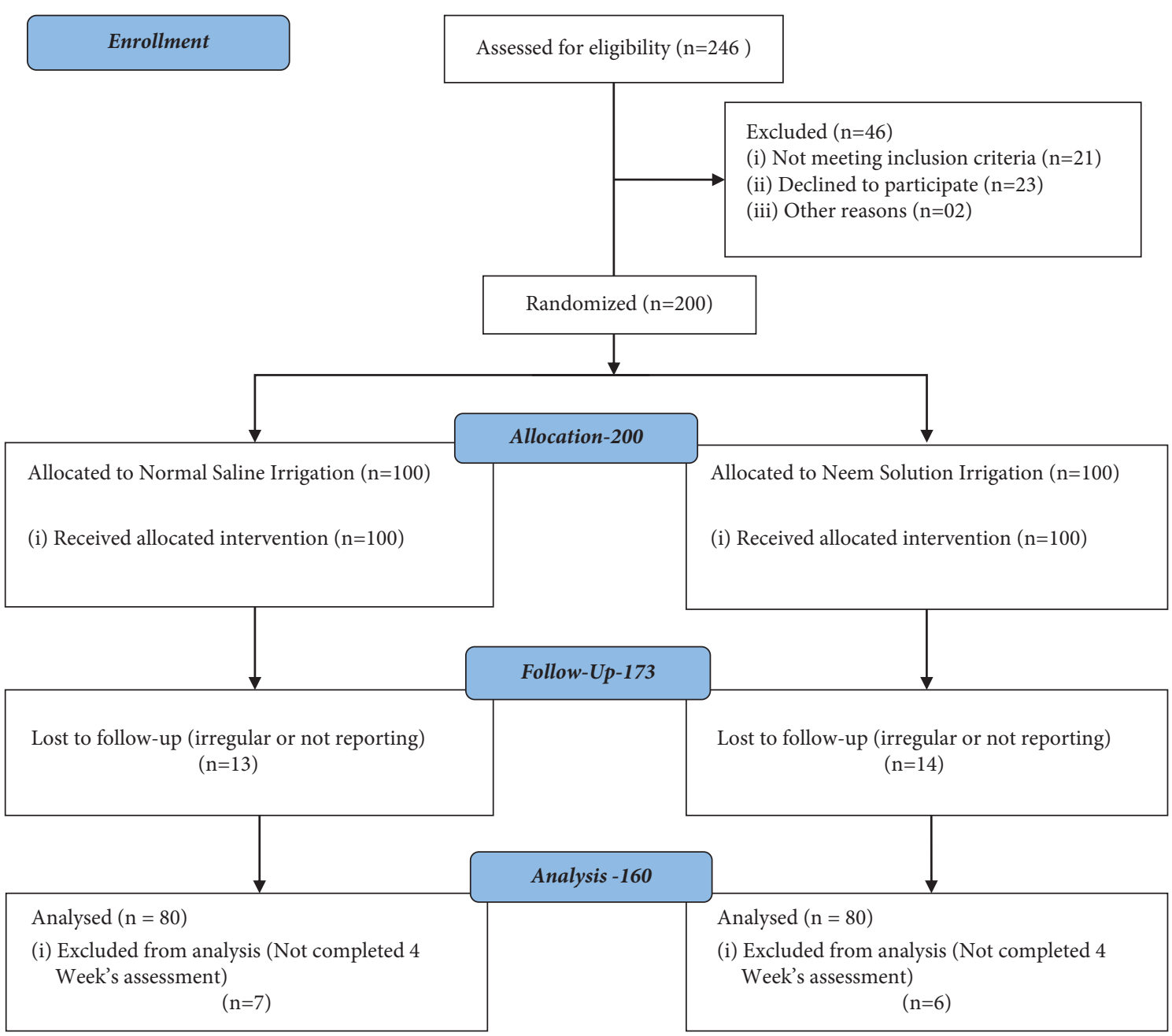

Figure 1: The Research Process

TABle 1: Demographic and clinical characteristics of diabetic foot ulcer individuals at baseline.

\begin{tabular}{|c|c|c|c|c|c|}
\hline \multirow[b]{2}{*}{ Characteristics } & \multicolumn{4}{|c|}{ Groups } & \multirow[b]{2}{*}{$P$} \\
\hline & Classification & $\begin{array}{c}\text { NS }(n=80) \\
n(\%)\end{array}$ & $\begin{array}{c}\mathrm{NE}(n=80) \\
n(\%)\end{array}$ & $x^{2}$ & \\
\hline \multirow{3}{*}{ Age (yr) } & $21-40$ & $4(5)$ & $9(11)$ & \multirow{3}{*}{2.281} & \multirow{3}{*}{0.320} \\
\hline & $41-60$ & $51(64)$ & $50(63)$ & & \\
\hline & $61-80$ & $25(31)$ & $21(26)$ & & \\
\hline \multirow{2}{*}{ Gender } & Male & $68(85)$ & $64(80)$ & \multirow{2}{*}{0.693} & \multirow{2}{*}{0.405} \\
\hline & Female & $12(15)$ & $16(20)$ & & \\
\hline \multirow{2}{*}{ Marital status } & Married & $78(98)$ & $78(98)$ & \multirow{2}{*}{0.000} & \multirow{2}{*}{1.000} \\
\hline & Single & $2(2)$ & $2(2)$ & & \\
\hline \multirow{4}{*}{ Education } & Postgraduate & $5(7)$ & $4(6)$ & \multirow{4}{*}{0.107} & \multirow{4}{*}{0.744} \\
\hline & Graduate & $23(29)$ & $19(24)$ & & \\
\hline & Secondary & $6(8)$ & $9(10)$ & & \\
\hline & Primary & $45(56)$ & $48(60)$ & & \\
\hline \multirow{2}{*}{ Occupation } & Employed & $58(73)$ & $59(74)$ & \multirow{2}{*}{0.032} & \multirow{2}{*}{0.858} \\
\hline & Unemployed & $22(27)$ & $21(27)$ & & \\
\hline \multirow{2}{*}{ Type of family } & Joint & $31(39)$ & $29(36)$ & \multirow{2}{*}{0.107} & \multirow{2}{*}{0.744} \\
\hline & Nuclear & $49(62)$ & $51(64)$ & & \\
\hline
\end{tabular}


TABLE 1: Continued.

\begin{tabular}{|c|c|c|c|c|c|}
\hline \multirow[b]{2}{*}{ Characteristics } & \multicolumn{5}{|c|}{ Groups } \\
\hline & Classification & $\begin{array}{c}\text { NS }(n=80) \\
n(\%)\end{array}$ & $\begin{array}{c}\mathrm{NE}(n=80) \\
n(\%)\end{array}$ & $x^{2}$ & $P$ \\
\hline \multirow{4}{*}{ Monthly income (Indian rupees) } & $<20,000$ & $31(39)$ & $47(59)$ & \multirow{4}{*}{6.831} & \multirow{4}{*}{0.77} \\
\hline & $21,000-40,000$ & $33(41)$ & $20(25)$ & & \\
\hline & $41,000-60,000$ & $14(17)$ & $11(13)$ & & \\
\hline & $61,000-80,000$ & $02(3)$ & $02(3)$ & & \\
\hline \multirow{4}{*}{ Duration of DM (years) } & $<5$ & $23(29)$ & $29(36)$ & \multirow{4}{*}{4.564} & \multirow{4}{*}{0.335} \\
\hline & $5-15$ & $26(32)$ & $28(35)$ & & \\
\hline & $15-25$ & $20(25)$ & $18(23)$ & & \\
\hline & $>25$ & $11(14)$ & $5(6)$ & & \\
\hline \multirow{2}{*}{ HbAlc level } & $\leq 7$ & $6(8)$ & $8(9)$ & \multirow{2}{*}{0.08} & \multirow{2}{*}{0.772} \\
\hline & $>7$ & $74(92)$ & $73(91)$ & & \\
\hline \multirow{2}{*}{ Vibration sensation } & Present & $34(43)$ & $36(45)$ & \multirow{2}{*}{0.626} & \multirow{2}{*}{0.744} \\
\hline & Absent & $46(57)$ & $44(55)$ & & \\
\hline
\end{tabular}

NS: normal saline; NE: neem leaf extract irrigation; DM: diabetes mellitus.

TABle 4: Results of RM-ANOVA of the PUSH score among the normal saline and neem leaf extract group.

\begin{tabular}{lcccr}
\hline Source of variation & df & ss & ms & $f$ \\
\hline Group factor & 1 & 553.816 & 553.816 & 20.278 \\
Time factor & 4 & 1690.558 & 422.640 & 221.685 \\
Interaction of the group and time & 4 & 52.651 & 13.163 & $<0.001$ \\
\hline
\end{tabular}

\section{References}

[1] M. Srinivasan Jayalakshmi, P. Thenmozhi, and R. Vijayaraghavan, "Plant Leaves Extract Irrigation on Wound Healing in Diabetic Foot Ulcers," Evidence-Based Complementary and Alternative Medicine, vol. 2021, Article ID 9924725, 9 pages, 2021. 